\title{
Coherent Quantum LQG Control *
}

\author{
Hendra I. Nurdin*, Matthew R. James*, and Ian R. Petersen ${ }^{\dagger}$ \\ \{Hendra.Nurdin,Matthew.James\}@anu.edu.au, i.petersen@adfa.edu.au \\ * Department of Engineering \\ The Australian National University \\ Canberra, ACT 0200, Australia \\ †School of Information Technology and Electrical Engineering \\ University of New South Wales at the Australian Defence Force Academy \\ Canberra, ACT 2600, Australia
}

\begin{abstract}
Based on a recently developed notion of physical realizability for quantum linear stochastic systems, we formulate a quantum LQG optimal control problem for quantum linear stochastic systems where the controller itself may also be a quantum system and the plant output signal can be fully quantum. Such a control scheme is often referred to in the quantum control literature as "coherent feedback control." It distinguishes the present work from previous works on the quantum LQG problem where measurement is performed on the plant and the measurement signals are used as input to a fully classical controller with no quantum degrees of freedom. The difference in our formulation is the presence of additional non-linear and linear constraints on the coefficients of the sought after controller, rendering the problem as a type of constrained controller design problem. Due to the presence of these constraints our problem is inherently computationally hard and this also distinguishes it in an important way from the standard LQG problem. We propose a numerical procedure for solving this problem based on an alternating projections algorithm and, as initial demonstration of the feasibility of this approach, we provide fully quantum controller design examples in which numerical solutions to the problem were successfully obtained. For comparison, we also consider the case of classical linear controllers that use direct or indirect measurements, and show that there exists a fully quantum linear controller which offers an improvement in performance over the classical ones.
\end{abstract}

Keywords: Quantum systems; Quantum control; Stochastic control; Linear quadratic regulators; Linear control systems

${ }^{*}$ This work was supported by the Australian Research Council. A preliminary version of this paper appeared in the Proceedings of the 17th IFAC World Congress, 2008. 


\section{Introduction}

Recent successes in quantum and nano-technology have provided a great impetus for research in the area of quantum feedback control systems; e.g. see [4, 35, 8, 2, 11]. It is reasonable to expect that quantum control is an area of research which could play a vital role towards realization of conceptual quantum information systems and quantum computers which are being extensively studied for potential benefits over their classical counterparts. One particular area in which significant theoretical and experimental advances have been achieved is quantum optics. In particular, linear quantum optics is one of the possible platforms being investigated for future communication systems (see [16, 20]) and quantum computers (see [18] and [26, Section 7.5]), besides being an area of independent interest in physics. Interestingly, under appropriate assumptions, the dynamics of some quantum optical devices can be approximately modeled by linear quantum stochastic differential equations driven by quantum Wiener processes; see [10]. For details on quantum stochastic differential equations and Wiener processes, see [15, 30, 6].

In general, quantum linear stochastic systems represented by linear Quantum Stochastic Differential Equations (QSDEs) with arbitrary constant coefficients need not correspond to physically meaningful systems. This is the same as for classical stochastic differential equations (throughout this paper we shall use the term "classical" to loosely refer to systems that have no quantum mechanical components). However, because classical linear stochastic systems can be implemented at least approximately, using analog or digital electronics, we regard them as always being realizable. Physical quantum systems must satisfy some additional constraints that restrict the allowable values for the system matrices defining the QSDEs. In particular, the laws of quantum mechanics dictate that closed quantum systems evolve unitarily, implying that (in the Heisenberg picture) certain canonical observables satisfy the so-called canonical commutation relations (CCR) at all times. Therefore, to characterize physically meaningful systems, [17] has introduced a formal notion of physically realizable quantum linear stochastic systems and derives a pair of necessary and sufficient characterizations for such systems in terms of constraints on their system matrices.

In this work, we build on the ideas in [17] and [32] and formulate a quantum LQG optimal control problem for quantum linear stochastic systems. The distinguishing feature of our work compared to previous treatments of the quantum LQG problem in the literature is that we allow the controller to be another quantum system whereas previous works only consider the case where the controller is a classical system driven by the result of continuous measurements performed on the output of the quantum plant. In the physics literature, (feedback) control using a fully quantum system is often referred to as "coherent feedback control" to distinguish it from control using a classical controller. Coherent controllers are of interest for, among other things, their potential for providing faster speed of processing/higher bandwidth (by dispensing of the use of "slow" electronics) and better performance (as will be demonstrated later in this paper). We stress that the coherent LQG controller design considered herein leads to a more difficult problem which cannot be solved using the usual approach of quantum conditioning and dynamic 
programming. By viewing the problem as a polynomial matrix programming problem, we show that by utilizing a non-linear change of variables due to [31], the problem can be systematically converted to a rank constrained LMI problem. To demonstrate the feasibility of numerically solving this problem, we provide a design example of stabilization of a quantum plant for which a solution to the rank constrained LMI problem was successfully obtained using an alternating projections algorithm due to [29].

The organization of the paper is as follows. We begin in Section 2 with an overview of quantum linear stochastic systems that are of interest in quantum optics. Section 3 then recalls some key definitions and results from [17] on physically realizable systems. In Section 4, we formulate a novel quantum LQG problem that allows the controller to be another quantum system. A numerical procedure for solving the quantum LQG problem is then proposed in Sections 5 and 6. In Section 7, we discuss an extension of the methodology developed in the preceding two sections. In Section 8, we consider the design of a fully quantum LQG controller for stabilizing a marginally stable quantum plant and show that there exists a fully quantum controller which offers an improved level of performance over a fully classical linear controller which uses direct or indirect measurements. Finally, in Section 9 we offer some concluding remarks.

\section{General quantum linear stochastic models in quan- tum optics}

We follow the quantum probabilistic setup of [17, Section II] and recall the notion of physical realizability introduced therein. To this end, consider linear non-commutative stochastic systems of the form

$$
\begin{aligned}
& d x(t)=A x(t) d t+B d w(t) ; \quad x(0)=x_{0} \\
& d y(t)=C x(t) d t+D d w(t)
\end{aligned}
$$

where $A, B, C$ and $D$ are real matrices in $\mathbb{R}^{n \times n}, \mathbb{R}^{n \times n_{w}}, \mathbb{R}^{n_{y} \times n}$ and $\mathbb{R}^{n_{y} \times n_{w}}$ respectively. Also, $\left(n, n_{w}, n_{y}\right.$ are positive integers), and $x(t)=\left[x_{1}(t) \ldots x_{n}(t)\right]^{T}$ is a vector of self-adjoint possibly non-commutative system variables.

The initial system variables $x(0)=x_{0}$ are Gaussian with state $f^{1}$, and satisfy the commutation relation 2

$$
\left[x_{j}(0), x_{k}(0)\right]=2 i \Theta_{j k}, \quad j, k=1, \ldots, n,
$$

where $\Theta$ is a real antisymmetric matrix with components $\Theta_{j k}$, and $i=\sqrt{-1}$. Here, the commutator is defined by $[A, B]=A B-B A$. To simplify matters and without loss of generality, we take the matrix $\Theta$ to be of one of the following forms:

\footnotetext{
${ }^{1}$ That is, $\operatorname{Tr}\left(\rho e^{i \lambda^{T} x_{0}}\right)=e^{i \lambda^{T} m-\frac{1}{2} \lambda^{T} G \lambda}$ for all $\lambda \in \mathbb{R}^{n}$ where $m \in \mathbb{R}^{n}$ and $G$ is a real symmetric matrix satisfying $G+i \Theta \geq 0$ with $\Theta$ as given in the text above; see, e.g., [22, 10, 30]).

${ }^{2}$ In the case of a single degree of freedom quantum particle, $x=\left(x_{1}, x_{2}\right)^{T}$ where $x_{1}=q$ is the position operator, and $x_{2}=p$ is the momentum operator. The annihilation operator is $a=(q+i p) / 2$. The commutation relations are $\left[a, a^{*}\right]=1$, or $[q, p]=2 i$.
} 
- Canonical if $\Theta=\operatorname{diag}(J, J, \ldots, J)$, or

- Degenerate canonical if $\Theta=\operatorname{diag}\left(0_{n^{\prime} \times n^{\prime}}, J, \ldots, J\right)$, where $0<n^{\prime} \leq n$.

Here, $J$ denotes the real skew-symmetric $2 \times 2$ matrix

$$
J=\left[\begin{array}{cc}
0 & 1 \\
-1 & 0
\end{array}\right],
$$

and the "diag" notation indicates a block diagonal matrix assembled from the given entries. To illustrate, the case of a system with one classical variable and two conjugate quantum variables is characterized by $\Theta=\operatorname{diag}(0, J)$, which is degenerate canonical. The vector quantity $w$ describes the input signals and is assumed to admit the decomposition

$$
d w(t)=\beta_{w}(t) d t+d \tilde{w}(t)
$$

where $\tilde{w}(t)$ is the noise part of $w(t)$ and $\beta_{w}(t)$ is a self adjoint, adapted process (see [15, 30, 6] for a discussion of adapted quantum processes). The process $\beta_{w}(t)$ serves to represent variables of other systems that may be passed to the system (1) via a connection. It is also represented on the same quantum probability space, which can be enlarged if necessary. Consequently, we assume that components of $\beta_{w}(t)$ commute with those of $d w(t)$. Furthermore, we will also assume that components of $\beta_{w}(t)$ commute with those of $x(t)$; this will simplify matters for the present work. The noise $\tilde{w}(t)$ is a vector of self-adjoint quantum noises with Ito table

$$
d \tilde{w}(t) d \tilde{w}^{T}(t)=F_{\tilde{w}} d t,
$$

where $F_{\tilde{w}}$ is a non-negative Hermitian matrix; e.g., see [30, 5]. This determines the following commutation relations for the noise components:

$$
\left[d \tilde{w}(t), d \tilde{w}^{T}(t)\right]=d \tilde{w}(t) d \tilde{w}^{T}(t)-\left(d \tilde{w}(t) d \tilde{w}^{T}(t)\right)^{T}=2 T_{\tilde{w}} d t,
$$

where we use the notation $S_{\tilde{w}}=\frac{1}{2}\left(F_{\tilde{w}}+F_{\tilde{w}}^{T}\right), \quad T_{\tilde{w}}=\frac{1}{2}\left(F_{\tilde{w}}-F_{\tilde{w}}^{T}\right)$ so that $F_{\tilde{w}}=S_{\tilde{w}}+T_{\tilde{w}}$. For instance, $F_{\tilde{w}}=\operatorname{diag}(1, I+i J)$ describes a noise vector with one classical component and a pair of conjugate quantum noises. (Here $I$ is the $2 \times 2$ identity matrix.) The noise processes can be represented as operators on Fock spaces; e.g., see [15, 30].

For simplicity, we also adopt the conventions of [17] to put the system (1) into a standard form. Therefore, we assume that in (1): (i) $n_{y}$ is even, and (ii) $n_{w} \geq n_{y}$. Furthermore, we also assume that $F_{\tilde{w}}$ is of the canonical form $F_{\tilde{w}}=I+i \operatorname{diag}(J, \ldots, J)$. Hence $n_{w}$ has to be even. Note that if $F_{\tilde{w}}$ is not canonical but of the form $F_{\tilde{w}}=I+$ $i \operatorname{diag}\left(0_{n^{\prime} \times n^{\prime}}, \operatorname{diag}(J, \ldots, J)\right)$ with $n^{\prime} \geq 1$, we may enlarge $w(t)$ (and hence also $\tilde{w}(t)$ ) and $B$ as before such that the enlarged noise vector, say $\tilde{w}^{\prime}$, can be taken to have an Ito matrix $F_{\tilde{w}^{\prime}}$ which is canonical. 


\section{Physical realizability of linear QSDEs}

A linear quantum stochastic system (1) with arbitrary system matrices need not represent any meaningful physical system. For example, quantum mechanics dictates that closed physical quantum systems evolve in a unitary manner which (in the Heisenberg or interaction picture) implies the preservation the canonical commutation relations (CCR): $x(t) x(t)^{T}-\left(x(t) x(t)^{T}\right)^{T}=2 i \Theta$ for all $t \geq 0$. This essentially restricts the allowable coefficients $A, B, C, D$ for a physically realizable quantum linear stochastic system. For this reason, [17] develops a precise notion of physical realizability based around the concept of an open quantum harmonic oscillator [17, Section 2] as the basic "dynamical unit" of a physically realizable quantum system. Such an oscillator is completely described by

a quadratic Hamiltonian $H=\frac{1}{2} x(0)^{T} R x(0)$, where $R$ is a real symmetric matrix, and a linear coupling operator $L=\Lambda x(0)$, where $\Lambda$ is a complex matrix (here we correct a minor error in [17, Definition 3.1] where the $\frac{1}{2}$ factor had been omitted from the definition of $H)$. We now give a brief summary of the main ideas developed in [17].

In the case that the system is fully quantum (i.e. $\Theta$ is canonical) then (1) is said to be physically realizable if it represents the dynamics of some open quantum harmonic oscillator [17, Section III-A]. If $\Theta$ is not canonical then a classical component of $x(t)$, say $x_{i}(t)$, is viewed as one component of a pair of (fictitious) canonically conjugate operators $\left(x_{i}(t), z_{i}(t)\right)$ satisfying $\left[x_{i}(t), z_{i}(t)\right]=2 i$ and which commute with all other components of $x(t)$. In that case (1) is said to be physically realizable if it can be embedded in some larger system also of the form (1) with a special structure (referred to as an augmentation of (1) in [17, Section III-B]) which is itself physically realizable, i.e. the larger system represents the dynamics of an open quantum harmonic oscillator. Based on this definition, [17] derives a pair of necessary and sufficient conditions for a quantum linear stochastic system of the form (1) to be physically realizable in terms of the system matrices, regardless of whether $\Theta$ is canonical or degenerate canonical. It is as follows, with $N_{y}=n_{y} / 2, N_{w}=n_{w} / 2$ and using the notation $\operatorname{diag}_{m}(K)$ to denote a block diagonal matrix with a square matrix $K$ appearing $m$ times on its diagonal blocks:

Theorem 1 ([17]) The system (1) is physically realizable if and only if:

$$
\begin{aligned}
i A \Theta+i \Theta A^{T}+B T_{w} B^{T} & =0 \\
B\left[\begin{array}{c}
I_{n_{y} \times n_{y}} \\
0_{\left(n_{w}-n_{y}\right) \times n_{y}}
\end{array}\right] & =\Theta C^{T} \operatorname{diag}_{N_{y}}(J),
\end{aligned}
$$

and $D=\left[\begin{array}{ll}I_{n_{y} \times n_{y}} & 0_{n_{y} \times\left(n_{w}-n_{y}\right)}\end{array}\right]$. Moreover for canonical $\Theta$, the corresponding Hamiltonian and coupling matrices have explicit expressions as follows. The Hamiltonian matrix $R$ is uniquely given by $R=\frac{1}{4}\left(-\Theta A+A^{T} \Theta\right)$, and the coupling matrix $\Lambda$ is given uniquely by

$$
\Lambda=-\frac{1}{2} i\left[\begin{array}{ll}
0_{N_{w} \times N_{w}} & I_{N_{w} \times N_{w}}
\end{array}\right]\left(\Gamma^{-1}\right)^{T} B^{T} \Theta
$$


where $\Gamma=P_{N_{w}} \operatorname{diag}_{N_{w}}(M)$ with $M=\frac{1}{2}\left[\begin{array}{cc}1 & i \\ 1 & -i\end{array}\right]$ and $P_{N_{w}}$ is a permutation matrix acting as $P_{N_{w}}\left(a_{1}, a_{2}, \ldots, a_{2 N_{w}}\right)^{T}=\left(a_{1}, a_{3}, \ldots, a_{2 N_{w}-1}, a_{2}, a_{4}, \ldots, a_{2 N_{w}}\right)^{T}$. In the case that $\Theta$ is degenerate canonical, a physically realizable augmentation of the system can be constructed to determine the Hamiltonian and coupling operators using the explicit formulas above.

Note that background information concerning the Hamiltonian and coupling matrices in quantum systems can be found in references [9, [10, 17].

\section{Formulation of the quantum LQG problem}

We consider plants described by non-commutative stochastic models of the following form:

$$
\begin{aligned}
d x(t) & =A x(t) d t+B d u(t)+B_{w} d w(t) ; \quad x(0)=x \\
d y(t) & =C x(t) d t+D_{w} d w(t) \\
z(t) & =C_{z} x(t)+D_{z} \beta_{u}(t) .
\end{aligned}
$$

Here $x(t)$ is a vector of plant variables, $w(t)$ is a quantum Wiener disturbance vector, $\beta_{u}(t)$ is an adapted, self-adjoint process commuting with $x(t)$ (i.e. $\beta_{u}(t) x(t)^{T}-\left(x(t) \beta_{u}(t)^{T}\right)^{T}=$ $0)$, and $u(t)$ is a control input of the form

$$
d u(t)=\beta_{u}(t) d t+d \tilde{u}(t)
$$

where $\beta_{u}(t)$ is the "signal part" and $\tilde{u}(t)$ is the noise part of $u(t)$. The vectors $w(t)$ and $\tilde{u}(t)$ are independent quantum noises (meaning that they live on distinct Fock spaces) with Ito matrices $F_{w}$ and $F_{\tilde{u}}$ that are all non-negative Hermitian. We also assume that $x(0) x(0)^{T}-\left(x(0) x(0)^{T}\right)^{T}=\Theta$.

Controllers are assumed to be non-commutative stochastic systems of the form

$$
\begin{aligned}
d \xi(t) & =A_{K} \xi(t) d t+B_{K 1} d w_{K 1}(t)+B_{K 2} d w_{K 2}(t)+B_{K 3} d y(t) \\
d u(t) & =C_{K} \xi(t) d t+d w_{K 1}(t)
\end{aligned}
$$

where $\xi(t)=\left[\begin{array}{llll}\xi_{1}(t) & \ldots & \xi_{n_{K}}(t)\end{array}\right]^{T}$ is a vector of self-adjoint controller variables of the same dimension as $x(t)$ (i.e. the controller is of the same order as the plant), $B_{K 2}$ is a square matrix of the same dimension as $A_{K}$, and $B_{K 1}$ has the same number of columns as there are rows of $C_{K}$. The noises $w_{K i}(t), i=1,2$, are vectors of non-commutative Wiener processes (in vacuum states) with non-zero Ito products and which are independent of $w(t)$. We assume that $\xi(0) \xi(0)^{T}-\left(\xi(0) \xi(0)^{T}\right)^{T}=\Theta_{K}$. Here $\Theta_{K}$ is the skew-symmetric commutation matrix for the controller variables $\xi$ that could be of a canonical or degenerate canonical form (cf. Section 2).

Assume further that $x(0) \xi(0)^{T}-\left(\xi(0) x(0)^{T}\right)^{T}=0$, i.e. the plant and controller are initially decoupled. The closed loop system is obtained by the identification $\beta_{u}(t) \equiv$ 
$C_{K} \xi(t)$ and $\tilde{u}(t) \equiv w_{K 1}(t)$, and interconnecting (9) and (11) to give

$$
\begin{aligned}
d \eta(t) & =\mathcal{A} \eta(t) d t+\mathcal{B} d w_{c l}(t) \\
z(t) & =\mathcal{C} \eta(t)
\end{aligned}
$$

where $\eta(t)=\left[\begin{array}{ll}x(t)^{T} & \xi(t)^{T}\end{array}\right]^{T}$,

$$
\begin{aligned}
w_{c l}(t) & =\left[\begin{array}{l}
w(t) \\
w_{K 1}(t) \\
w_{K 2}(t)
\end{array}\right] ; \mathcal{A}=\left[\begin{array}{ll}
A & B C_{K} \\
B_{K 3} C & A_{K}
\end{array}\right] ; \\
\mathcal{B} & =\left[\begin{array}{lll}
B_{w} & B & 0_{2 \times 2} \\
B_{K 3} D_{w} & B_{K 1} & B_{K 2}
\end{array}\right] ; \quad \mathcal{C}=\left[\begin{array}{ll}
C_{z} & D_{z} C_{K}
\end{array}\right] .
\end{aligned}
$$

With 12 we associate a quadratic performance index

$$
J\left(t_{f}\right)=\int_{0}^{t_{f}}\left\langle z^{T}(t) z(t)\right\rangle d t .
$$

Here the notation $\langle\cdot\rangle$ is standard and refers to quantum expectation (e.g., see [24]). In this case, the quantum expectation is on the composite system (plant, controller and all quantum noises) following [17].

We shall proceed to derive an explicit expression for this performance index; see also [32]. To this end, define the symmetrized covariance matrix $P(t)$ by

$$
P(t)=\frac{1}{2}\left\langle\eta(t) \eta^{T}(t)+\left(\eta(t) \eta^{T}(t)\right)^{T}\right\rangle .
$$

Using the quantum Ito rule, we have

$$
\begin{aligned}
d P(t)= & \frac{1}{2}\left(\left\langle d \eta(t) \eta^{T}(t)\right\rangle+\left\langle\left(d \eta(t) \eta^{T}(t)\right)^{T}\right\rangle+\left\langle\eta(t) d \eta^{T}(t)\right\rangle+\right. \\
& \left.\left\langle\left(\eta(t) d \eta^{T}(t)\right)^{T}\right\rangle+\left(\mathcal{B} F_{w_{c l}} \mathcal{B}^{T}+\left(\mathcal{B} F_{w_{c l}} \mathcal{B}^{T}\right)^{T}\right) d t\right) \\
= & \left(\mathcal{A} P(t)+P(t) \mathcal{A}^{T}+\frac{1}{2} \mathcal{B}\left(F_{w_{c l}}+F_{w_{c l}}^{T}\right) \mathcal{B}^{T}\right) d t \\
= & \left(\mathcal{A P}(t)+P(t) \mathcal{A}^{T}+\mathcal{B} \mathcal{B}^{T}\right) d t
\end{aligned}
$$

where the last equality follows from our convention that all noises are canonical (hence $\left.\frac{1}{2}\left(F_{w_{c l}}+F_{w_{c l}}^{T}\right)=I\right)$. Hence $P(\cdot)$ satisfies the differential equation

$$
\dot{P}(t)=\mathcal{A} P(t)+P(t) \mathcal{A}^{T}+\mathcal{B B}^{T} ; \quad P(0)=P_{0} .
$$

We now have, using the symmetry of $\mathcal{C}^{T} \mathcal{C}$ and $P$, that

$$
\begin{aligned}
\left\langle z^{T} z\right\rangle & =\left\langle\eta^{T} \mathcal{C}^{T} \mathcal{C} \eta\right\rangle \\
& =\left\langle\operatorname{Tr}\left(\eta^{T} \mathcal{C}^{T} \mathcal{C} \eta\right)\right\rangle \\
& =\frac{1}{2}\left\langle\operatorname{Tr}\left(\mathcal{C}^{T} \mathcal{C}\left[\eta \eta^{T}+\left(\eta \eta^{T}\right)^{T}\right]\right)\right\rangle \\
& =\operatorname{Tr}\left(\mathcal{C}^{T} \mathcal{C} P\right)
\end{aligned}
$$


Hence, the performance index (13) can be expressed as

$$
J\left(t_{f}\right)=\int_{0}^{t_{f}} \operatorname{Tr}\left(\mathcal{C}^{T} \mathcal{C} P(t)\right) d t
$$

where $P(t)$ solves $(15)$. We will focus our attention on the infinite horizon case where we allow $t_{f} \uparrow \infty$. Assuming that $\mathcal{A}$ is asymptotically stable, standard results on Lyapunov equations give us $\lim _{t \rightarrow \infty} P(t)=P$, where $P$ is the unique symmetric positive definite solution of the Lyapunov equation:

$$
\mathcal{A P}+P \mathcal{A}^{T}+\mathcal{B B}^{T}=0
$$

Furthermore, by standard methods of analysis we have

$$
\limsup _{t_{f} \rightarrow \infty} \frac{1}{t_{f}} \int_{0}^{t_{f}}\left\langle z^{T}(t) z(t)\right\rangle d t=\operatorname{Tr}\left(\mathcal{C}^{T} \mathcal{C} P\right)=\operatorname{Tr}\left(\mathcal{C} P \mathcal{C}^{T}\right) .
$$

As before, let $\operatorname{diag}_{m}(J)$ denote a block diagonal $2 m \times 2 m$ matrix with $m J$ matrices on the diagonal blocks and let $n_{i}$ denote the dimension of $w_{K i}$ for $i=1,2,3$. We may now formulate our quantum LQG control problem for an infinite horizon as follows:

Problem 2 (Quantum LQG synthesis) Given a fixed choice of $\Theta_{K}$, find controller matrices $A_{K}, B_{K 1}, B_{K 2}, B_{K 3}$ and $C_{K}$ that minimizes the cost functional $J_{\infty}=\operatorname{Tr}\left(\mathcal{C} P \mathcal{C}^{T}\right)$ subject to the constraint that the controller (11) is physically realizable. That is, $A_{K}$, $B_{K 1}, B_{K 2}, B_{K 3}, C_{K}$ satisfy the conditions of Theorem 1 with the identification: $A \equiv A_{K}$, $B=\left[\begin{array}{lll}B_{K 1} & B_{K 2} & B_{K 3}\end{array}\right], C \equiv C_{K}, D \equiv\left[\begin{array}{ll}I_{n_{u} \times n_{u}} & 0\end{array}\right]$, and $w \equiv\left[\begin{array}{lll}w_{K 1}^{T} & w_{K 2}^{T} & y^{T}\end{array}\right]^{T}$, leading to the pair of constraints:

$$
\begin{aligned}
& A_{K} \Theta_{K}+\Theta_{K} A_{K}^{T}+B_{K 1} \operatorname{diag}_{n_{1} / 2}(J) B_{K 1}^{T}+B_{K 2} \operatorname{diag}_{n_{2} / 2}(J) B_{K 2}^{T}+ \\
& \quad B_{K 3} \operatorname{diag}_{n_{3} / 2}(J) B_{K 3}^{T}=0 \\
& B_{K 1}=\Theta_{K} C_{K}^{T} \operatorname{diag}_{n_{u} / 2}(J) .
\end{aligned}
$$

In the above problem $\Theta_{K}$ is a fixed but freely specified parameter that determines the type of controller sought. For example, if $\Theta_{K}$ is canonical then the controller will be fully quantum. Our formulation of the quantum LQG problem differs from previous formulations of the quantum LQG problem, such as given in [9] and [8]. The important difference is that in the earlier works, the controller is classical whereas in our formulation we seek a controller which may possibly be another quantum system (depending on how $\Theta_{K}$ is defined) which generates an optical field to drive the quantum plant. What is new in the formulation are the additional constraints (18) and $(19)$ that must also be satisfied by the controller to be physically realizable. This is natural since for real applications, the controller should represent a physical system. The constraint (18) is a non-convex, non-linear equality constraint on the controller matrices $A_{K}, B_{K 1}, B_{K 2}, B_{K 3}$ and $C_{K}$ that presents a formidable challenge in the controller design. 
At present we do not know if there exists an exact or analytical solution to Problem 2. Moreover, in our experience this non-convex problem is difficult to solve numerically using a general purpose optimizer such as the 'fmincon' routine in the Matlab Optimization Toolbox; see [34]. In our investigation, it has been more fruitful to consider the relaxed problem of finding a controller that achieves the cost bound $J_{\infty}<\gamma$ for some pre-specified bound $\gamma>0$, and to reformulate this problem into a rank constrained LMI feasibility problem. As in $H^{\infty}$ synthesis, a solution to Problem 2 can, in principle, be found iteratively by employing a bisection method, or a variant thereof, on the bound $\gamma$. Therefore, we focus our attention instead on the following problem:

Problem 3 Given a fixed choice of $\Theta_{K}$ and cost bound parameter $\gamma>0$, find controller matrices $A_{K}, B_{K 1}, B_{K 2}, B_{K 3}$ and $C_{K}$ such that the following conditions hold.

F1. There exists a symmetric matrix $P>0$ satisfying $(1 \%)$.

F2. $J_{\infty}=\operatorname{Tr}\left(\mathcal{C P C}^{T}\right)<\gamma$.

F3. The physical realizability constraints (18) and (19) are satisfied.

\section{Reformulation of the quantum LQG problem into a rank constrained LMI problem}

We shall now discuss how to transform Problem 3 into a rank constrained LMI problem which is amenable to numerical methods. To best illustrate the idea, we opt to restrict our attention to the case where $\Theta_{K}$ is canonical. Moreover, to facilitate easy and explicit exposition of the matrix lifting and linearization technique, we shall take for a "canonical" example, a plant and controller of order $n$ (recall that in our setup we are looking for a controller which is of the same order as the plant) with $n_{y}=n_{u}=n$ and $B_{K 1}, B_{K 2}, B_{K 3}$, $C_{K}$ all of dimension $n \times n$. Nonetheless, the matrix lifting principle described for this canonical case can in principle be adapted to more general scenarios and for the case in which $\Theta_{K}$ is degenerate canonical, that is, the case where the controller has both quantum and classical degrees of freedom. However, the lifting is not necessarily unique and is too complicated to describe in a general form. More importantly, for efficiency the choice of suitable lifting variables should in any case be considered on a case by case basis to exploit any existing structure in a particular problem.

Consider a $n$-th order plant (9) with $n_{y}=n_{u}=n$ and a $n$-th order controller (11) with $n_{w_{K 1}}=n_{w_{K 2}}=n$ (hence $B_{K 1}, B_{K 2} \in \mathbb{R}^{n \times n}$ ). Then $P$ will be a symmetric matrix of dimension $2 n \times 2 n$. The first step is to transform the constraints (17) and $J_{\infty}<\gamma$ into an LMI constraint. To do this we exploit a non-linear change of variables given in [31, Eq.(35)], but to do this we first need to suitably redefine our plant and controller equations while leaving the closed-loop equations unaltered. To this end, let us redefine 
our plant as:

$$
\begin{aligned}
d x(t) & =A x(t) d t+B \beta_{u}(t)+B_{w^{\prime}}^{\prime} d w^{\prime}(t) ; \quad x(0)=x \\
d y^{\prime}(t) & =C^{\prime} x(t) d t+D_{w^{\prime}}^{\prime} d w^{\prime}(t) \\
z(t) & =C_{z} x(t)+D_{z} \beta_{u}(t)
\end{aligned}
$$

with $w^{\prime}=\left[\begin{array}{lll}w^{T} & w_{K 1}^{T} & w_{K 2}^{T}\end{array}\right]^{T}, B_{w^{\prime}}^{\prime}=\left[\begin{array}{lll}B_{w} & B & 0_{n \times n}\end{array}\right], C^{\prime}=\left[\begin{array}{lll}0_{n \times n} & 0_{n \times n} & C^{T}\end{array}\right]^{T}$ and

$$
D_{w^{\prime}}^{\prime}=\left[\begin{array}{ccc}
0_{n \times n_{w}} & I_{n \times n} & 0_{n \times n} \\
0_{n \times n_{w}} & 0_{n \times n_{w}} & I_{n \times n} \\
D_{w} & 0_{n \times n} & 0_{n \times n}
\end{array}\right] .
$$

Here $y^{\prime}$ is the output equation for the modified plant that now includes the quantum noise $w_{K 2}$ that enters in the controller, but not in the original plant. In this way all noises can now be thought of as coming from the modified plant, as in the setup of standard classical LQG problems. Then, we also redefine our controller equations as:

$$
\begin{aligned}
d \xi(t) & =A_{K} \xi(t) d t+B_{K} d y^{\prime}(t) \\
\beta_{u}(t) & =C_{K} \xi(t)
\end{aligned}
$$

with $B_{K}=\left[\begin{array}{lll}B_{K 1} & B_{K 2} & B_{K 3}\end{array}\right]$. It is easily seen that interconnecting (20) and (21) gives the closed-loop equation (12). Now we are in the setup of [31] with $D_{K}=0$ in [31, Eq.(2)].

We now follow [31] by introducing auxiliary variables $N, M, \mathbf{X}, \mathbf{Y}, Q \in \mathbb{R}^{n \times n}$, with $\mathbf{X}, \mathbf{Y}, Q$ symmetric, and applying the following non-linear change of variables (see [31, Section IV-B] with $\left.\hat{\mathbf{D}}=D_{K}=0\right)$ :

$$
\begin{aligned}
& \mathbf{A}=N A_{K} M^{T}+N B_{K} C^{\prime} \mathbf{X}+\mathbf{Y} B C_{K} M^{T}+Y A \mathbf{X} \\
& \mathbf{B}=N B_{K} ; \\
& \mathbf{C}=C_{K} M^{T} .
\end{aligned}
$$

Then, the constraints (17) and $J_{\infty}<\gamma$ can be rewritten as the LMI constraint [31, Eq.(14)]:

$$
\begin{aligned}
& {\left[A \mathbf{X}+\mathbf{X} A^{T}+B \mathbf{C}+(B \mathbf{C})^{T} \quad \mathbf{A}^{T}+A\right.} \\
& \mathbf{A}+A^{T} \quad A^{T} \mathbf{Y}+\mathbf{Y} A+\mathbf{B} C^{\prime}+\left(\mathbf{B} C^{\prime}\right)^{T} \\
& \left(B_{w^{\prime}}^{\prime}\right)^{T} \quad\left(\mathbf{Y} B_{w^{\prime}}^{\prime}+\mathbf{B} D_{w^{\prime}}^{\prime}\right)^{T} \\
& \left.\begin{array}{c}
B_{w^{\prime}}^{\prime} \\
\mathbf{Y} B_{w^{\prime}}^{\prime}+\mathbf{B} D_{w^{\prime}}^{\prime} \\
-I
\end{array}\right]<0 \\
& {\left[\begin{array}{ccc}
\mathbf{X} & I & \left(C_{z} \mathbf{X}+D_{z} \mathbf{C}\right)^{T} \\
I & \mathbf{Y} & C_{z}^{T} \\
C_{z} \mathbf{X}+D_{z} \mathbf{C} & C_{z} & Q
\end{array}\right]>0} \\
& \operatorname{Tr}(Q)<\gamma \text {. }
\end{aligned}
$$


Since the controller is of the same order as the plant, the matrices $N$ and $M$ can be freely chosen to be any pair of (invertible) square matrices satisfying $M N^{T}=I-\mathrm{XY}$.

Once matrices A, B, C, X, Y, $Q$ satisfying the LMIs (25)-27) and matrices $N$ and $M$ satisfying the conditions of the last paragraph have been found, the original controller matrices $A_{K}, B_{K}, C_{K}$ can be reconstructed as [31, Eq.(40)]:

$$
\begin{aligned}
& C_{K}=\mathbf{C} M^{-T} ; \\
& B_{K}=N^{-1} \mathbf{B} ; \\
& A_{K}=N^{-1}\left(\mathbf{A}-N B_{K} C^{\prime} \mathbf{X}-\mathbf{Y} B C_{K} M^{T}-\mathbf{Y} A \mathbf{X}\right) M^{-T} .
\end{aligned}
$$

Multiplying the left and right hand sides of 18 with $N$ and $N^{T}$, respectively, and introducing new variables $\breve{N}=N \Theta_{K}$ (keep in mind here that $\Theta_{K}$ is a fixed matrix), $\breve{A}_{K}=N A_{K}$ and $\breve{B}_{K i}=N B_{K i}, i=1,2,3,18$ and $(19)$ can be expressed as:

$$
\begin{aligned}
& \left(-\mathbf{A} M^{-T}+\left(\breve{B}_{K 3} C+\mathbf{Y} A\right) \mathbf{X} M^{-T}+\mathbf{Y} B C_{K}\right) \breve{N}^{T} \\
& \quad+\breve{N}\left(\mathbf{A} M^{-T}-\left(\breve{B}_{K 3} C+\mathbf{Y} A\right) \mathbf{X} M^{-T}-\mathbf{Y} B C_{K}\right)^{T}+ \\
& \quad \sum_{i=1}^{3} \breve{B}_{K i} \operatorname{diag}_{n / 2}(J) \breve{B}_{K i}^{T}=0 ; \\
& \breve{B}_{K 1}=\breve{N} C_{K}^{T} \operatorname{diag}_{n / 2}(J) .
\end{aligned}
$$

Conversely, if $A_{K}, B_{K 1}, B_{K 2}, B_{K 3}, C_{K}$ solve Problem 3 and $P$ is the solution of (17), then the LMIs (25)-(27) are all satisfied for some pair of square matrices $M$ and $N$ satisfying $M N^{T}=I-\mathbf{X Y}$; see [31, Section IV-B]. Furthermore, since the solution is physically realizable, (31) and (32) are also satisfied. We summarize the preceding discussion in the following theorem:

Theorem 4 Under the assumptions of this section, Problem 3 has a solution for a given $\gamma>0$ if and only if there exist matrices $\mathbf{A}, \breve{B}_{K 1}, \breve{B}_{K 2}, \breve{B}_{K 3}, \mathbf{C}, \mathbf{X}, \mathbf{Y}, \stackrel{N}{ }, M, N$, $C_{K}$ satisfying the LMIs (25)-(27) (with $\mathbf{B}=\left[\begin{array}{ccc}\breve{B}_{K 1} & \breve{B}_{K 2} & \breve{B}_{K 3}\end{array}\right]$ ) and the constraints (31)-(32), $\breve{N}=N \Theta_{K}, N M^{T}=I-\mathbf{Y X}$ and $\mathbf{C}=C_{K} M^{T}$.

Note that (31) and (32) are polynomial matrix equality constraints in the parameters $\left(\mathbf{A}, \breve{B}_{K 1}, \breve{B}_{K 2}, \breve{B}_{K 3}, \mathbf{C}, \mathbf{X}, \mathbf{Y}, \breve{N}, M^{-T}\right)$. By this we mean that they are equality constraints in a matrix-valued multivariate polynomial with matrix-valued variables. If we take as decision variables the elements of the parameters (for symmetric variables such as $\mathbf{X}$ we need only take the upper triangular elements) then this becomes a collection of scalar multivariate polynomial equalities. It is well known that by introducing additional variables, called lifting variables, and some auxiliary equality constraints, general polynomial equality and inequality constraints can be "linearized" and transformed into linear equality and inequality constraints in some symmetric positive semidefinite matrix $X$, plus a rank one constraint: $\operatorname{rank}(X)=1$ [25, 7]. However, converting polynomial matrix constraints into a collection of scalar polynomial constraints may not be desirable as the resulting 
scalar polynomials could be of orders much higher than the order of the original matrix polynomial. Subsequently, when there are many decision variables the resulting scalar polynomial program may easily become too large to handle numerically. This is true in our present case, if we are to scalarize (31) then we would end up with 34 decision variables and constraints in polynomials of order 4 , a substantially large problem. Therefore we would like to keep the matrix structure of our problem and try to find suitable matrix lifting variables instead. The idea is similar to the scalar version, but we need to take care of the fact that, unlike scalars, matrices do not in general commute with one another.

We now proceed to linearize (31) and (32) by introducing appropriate matrix lifting variables and the associated equality constraints, and transforming them into an LMI with a rank $n$ constraint. However, in what will follow we set $M=I_{n \times n}$ and $N=I-\mathbf{Y X}$; this removes one free matrix variable, namely $M^{-T}$, and reduces the complexity of the problem. The 14 matrix lifting variables $W_{1}, W_{2}, \ldots, W_{14} \in \mathbb{R}^{n \times n}$ are as follows: $W_{i}=\breve{B}_{K i} J$, $i=1,2,3, W_{4}=\mathbf{Y} B, W_{5}=\breve{B}_{K 3} C+\mathbf{Y} A, W_{6}=\breve{N C}^{T}, W_{7}=\breve{N} \mathbf{X}, W_{8}=\mathbf{A} \breve{N}^{T}, W_{9}=\mathbf{Y X}$, $W_{10}=W_{4} W_{6}^{T}, W_{11}=W_{5} W_{7}^{T}, W_{12}=W_{1} \breve{B}_{K 1}^{T}, W_{13}=W_{2} \breve{B}_{K 2}^{T}$ and $W_{14}=W_{3} \breve{B}_{K 3}^{T}$. Now, let $Z$ be a $23 n \times 23 n$ symmetric matrix,

$$
\begin{aligned}
\mathbf{Z}_{i, j} & =\left[Z_{k l}\right]_{k=i n+1,(i+1) n, l=j n+1,(j+1) n}, \\
x & =\left(x_{1}, \ldots, x_{8}\right)=(1,2, \ldots, 8),
\end{aligned}
$$

and

$$
v=\left(v_{1}, \ldots, v_{14}\right)=(9,10, \ldots, 22) .
$$

We require that $Z$ satisfy the constraints:

$$
\begin{array}{cc}
Z \geq 0 & \mathbf{Z}_{v_{6}, 1}-\mathbf{Z}_{x_{8}, x_{5}}=0 \\
\mathbf{Z}_{0,0}-\bar{I}_{n \times n}=0 & \mathbf{Z}_{v_{7}, 1}-\mathbf{Z}_{x_{8}, x_{6}}=0 \\
\mathbf{Z}_{1, x_{6}}-\mathbf{Z}_{x_{6}, 1}=0 & \mathbf{Z}_{v_{8}, 1}-\mathbf{Z}_{x_{1}, x_{8}}=0 \\
\mathbf{Z}_{1, x_{7}}-\mathbf{Z}_{x_{7}, 1}=0 & \mathbf{Z}_{v_{9}, 1}-\mathbf{Z}_{x_{7}, x_{6}}=0 \\
\mathbf{Z}_{v_{1}, 1}-\mathbf{Z}_{x_{2}, 1} \operatorname{diag}_{n / 2}(J)=0 & \mathbf{Z}_{v_{10}, 1}-\mathbf{Z}_{v_{4}, v_{6}}=0 \\
\mathbf{Z}_{v_{2}, 1}-\mathbf{Z}_{x_{3}, 1} \operatorname{diag}_{n / 2}(J)=0 & \mathbf{Z}_{v_{11}, 1}-\mathbf{Z}_{v_{5}, v_{7}}=0 \\
\mathbf{Z}_{v_{3}, 1}-\mathbf{Z}_{x_{4}, 1} \operatorname{diag}_{n / 2}(J)=0 & \mathbf{Z}_{v_{12}, 1}-\mathbf{Z}_{v_{1}, x_{2}}=0 \\
\mathbf{Z}_{v_{4}, 1}-\mathbf{Z}_{x_{7}, 1} B=0 & \mathbf{Z}_{v_{13}, 1}-\mathbf{Z}_{v_{2}, x_{3}}=0 \\
\mathbf{Z}_{v_{5}, 1}-\mathbf{Z}_{x_{4}, 1} C-\mathbf{Z}_{x_{7}, 1} A=0 & \mathbf{Z}_{v_{14}, 1}-\mathbf{Z}_{v_{3}, x_{4}}=0 \\
\mathbf{Z}_{x_{8}, 1}-\Theta_{K}+\mathbf{Z}_{v_{9}, 1} \Theta_{K}=0 . &
\end{array}
$$

Here, terms of the form $\mathbf{Z}_{a, b}$ with $a, b \in\left\{x_{1}, \ldots, x_{8}\right\} \cup\left\{v_{1}, \ldots, v_{14}\right\}$ should be identified with $\mathbf{Z}_{a, 1}\left(\mathbf{Z}_{b, 1}\right)^{T}$. The LMI constraints (25)-(27) can be expressed in terms of $Z$ by replacing $\mathbf{A}, \mathbf{B}, \mathbf{C}, \mathbf{X}, \mathbf{Y}$ respectively with

$\mathbf{Z}_{x_{1}, 1},\left[\begin{array}{lll}\mathbf{Z}_{x_{2}, 1} & \mathbf{Z}_{x_{3}, 1} & \mathbf{Z}_{x_{4}, 1}\end{array}\right], \mathbf{Z}_{x_{5}, 1}, \mathbf{Z}_{x_{6}, 1}, \mathbf{Z}_{x_{7}, 1}$, while the physical realizability constraints (31) and (32) become the following pair of linear equality constraints:

$$
\begin{aligned}
& -\mathbf{Z}_{v_{8}, 1}+\mathbf{Z}_{v_{8}, 1}^{T}+\mathbf{Z}_{v_{11}, 1}-\mathbf{Z}_{v_{11}, 1}^{T}+\mathbf{Z}_{v_{10}, 1}-\mathbf{Z}_{v_{10}, 1}^{T} \\
& \quad+\mathbf{Z}_{v_{12}, 1}+\mathbf{Z}_{v_{13}, 1}+\mathbf{Z}_{v_{14}, 1}=0 \\
& \mathbf{Z}_{x_{2}, 1}-\mathbf{Z}_{v_{6}, 1} \operatorname{diag}_{n / 2}(J)=0
\end{aligned}
$$


Finally, we also require that $Z$ satisfy a rank $n$ constraint:

$$
\operatorname{rank}(Z) \leq n
$$

To understand the above rank constrained LMI and its relation to our original constraints, suppose that there is a $Z$ satisfying (33)-(35) and the LMI constraints (expressed in terms of block elements of $Z$ ). Then, since $Z \geq 0$ and is of rank at most $n$, we may factorize it as $Z=V V^{T}$, where $V \in \mathbb{R}^{23 n \times n}$ and satisfies $\left[V_{i j}\right]_{i, j=1, n}=I_{n \times n}$, and by (33) we recover $\mathbf{A}, \breve{B}_{K i}(i=1,2,3), \mathbf{C}, \mathbf{X}, \mathbf{Y}, \breve{N}$ respectively as $\mathbf{Z}_{x_{1}, 1}, \ldots, \mathbf{Z}_{x_{8}, 1}$, and also recover $W_{i}=\mathbf{Z}_{v_{i}, 1}, i=1, \ldots, 14$. Then $N=\breve{N} \Theta_{K}^{-1}$ and $\mathbf{B}_{K i}=N^{-1} \breve{B}_{K i}(i=1,2,3)$. The controller matrices $A_{K}, B_{K}, C_{K}$ are given by (28)-(30) and by construction they will satisfy (25)-(27), (18) and (19). Thus, we obtain a solution to Problem 3.

It should be noted that due to the simplifying assumptions $M=I$ and $N=I-\mathbf{Y X}$, solvability of the rank constrained LMI problem formulated above is only sufficient for solvability of Problem 3 . In fact, in Theorem 4 it is only required that $M$ and $N$ satisfy $N M^{T}=I-\mathbf{Y X}$. Nonetheless, it is not difficult to see that by (i) introducing additional variables $M, N, C_{K} \equiv \mathbf{C} M^{-T}, \breve{\mathbf{A}} \equiv \mathbf{A} M^{-T}, \breve{\mathbf{X}} \equiv \mathbf{X} M^{-T}$, (ii) additional lifting variables $W_{15}=N M^{T}, W_{16}=\breve{\mathbf{A}} M^{T}, W_{17}=\breve{\mathbf{X}} M^{T}, W_{18}=C_{K} M^{T}$ and associated constraints $W_{15}-I+W_{9}=0, \mathbf{A}-W_{16}=0, \mathbf{X}-W_{17}=0, \breve{N}-N \Theta_{K}=0, \mathbf{C}-W_{18}=0$ (iii) redefining $W_{6}=\breve{N} C_{K}^{T}, W_{7}=\breve{N} \breve{\mathbf{X}}^{T}$ and $W_{8}=\breve{\mathbf{A}} \breve{N}^{T}$, and (iv) enlarging and redefining $Z$ as well as the set of constraints (33) and (34) accordingly, these simplifying assumptions can be removed to make the resulting rank constrained LMI problem also necessary for solvability of Problem 3 , but at the expense of having to solve a larger problem. The simplification we have proposed here, however, may be especially useful for reducing the complexity of problems with a plant dimension larger than 2. We conclude this section with the following remark.

Remark 5 In the formulation of this section, it is not actually essential to fix $\Theta_{K}$ to be $\operatorname{diag}_{n / 2}(J)$. Instead, it may also be fixed to be $\Theta_{K}^{S}=S \operatorname{diag}_{n / 2}(J) S^{T}$ for any real invertible matrix $S$. Indeed, if $A_{K}^{S}, B_{K i}^{S}(i=1,2,3), C_{K}^{S}$ solves Problem 3 for $\Theta_{K}=\Theta_{K}^{S}$ then $A_{K}=S^{-1} A_{K}^{S} S, B_{K i}=S^{-1} B_{K i}^{S},(i=1,2,3), C_{K}=C_{K}^{S} S$ solves Problem 3 for $\Theta_{K}=\operatorname{diag}_{n / 2}(J)$; for details, see a related discussion in Section 7 where $\Theta_{K}$ is allowed to be a free variable. This added flexibility will be valuable for numerical attempts at solving Problem 3 .

\section{Numerical solution of the rank constrained LMI problem}

We have seen in the preceding section that our problem is essentially a polynomial matrix programming (to be precise, feasibility) problem (since LMIs can themselves be viewed as polynomial matrix inequalities) and that the latter can be converted to a rank constrained 
problem. It is well-known that many important practical control problems can be formulated as polynomial programming problems, including reduced order robust controller design, static output feedback and gain scheduling (see [13] and the references therein). They are non-convex and non-linear problems that are, in general, difficult to solve. In fact, some of these problems are known to be NP-hard [33, 21].

If one tries to directly attack the (scalar or matrix) polynomial programming problem, then a specialized method for solving them is to employ LMI relaxations techniques based on the theory of moments and the dual theory of sum of squares (SOS) polynomials; see [21, 19, 14, 13. Under appropriate conditions, relaxation methods can be guaranteed to converge as the order of relaxation is increased and it can be checked whether a global optima may have been obtained at a particular relaxation. Despite its attractive features, the size of the relaxed LMI problem grows very quickly with the number of decision variables, the degree of polynomials involved and the order of relaxation, making the method impractical for problems with many decision variables.

On the other hand, if the problem is converted to a rank constrained LMI problem then there are iterative algorithms in the literature that try to directly search for a feasible point satisfying the set of LMIs and the rank constraint, mostly based on the idea of alternating projections (see [29] and the references therein). The main drawback of these algorithms is that they are difficult to analyze and are not in general guaranteed to converge from arbitrary starting points, even if a solution exists. However, since there are no relaxations involved that increase the size of the problem to be solved, they can be more attractive for solving medium and larger size polynomial programming problems. This makes them more suitable for our current problem, which can be considered to be of a substantial size (recall that if it is converted to a scalar polynomial programming problem then there would be 34 decision variables and involve multivariate polynomials of up to degree 4).

To solve the rank constrained LMI problem formulated in the last section, we shall use an algorithm by [29] that has been implemented in the freely available Matlab toolbox LMIRank (see [28]) and can be called via the Yalmip optimization prototyping environment; see [23]. This algorithm is also based on alternating projections but, unlike previous alternating projections algorithms, has a built-in Newton step that has the potential to accelerate convergence. In Section 8, we will use this method to numerically solve an example coherent LQG control problem.

As mentioned earlier, solvers for rank constrained LMI problems are not guaranteed to converge from arbitrary starting points. Therefore, it is important to have a heuristic method for choosing starting points for these algorithms. For a given $\gamma>0$, to obtain a starting point for the LMIRank solver we suggest to first solve the LMIs (25)-(27) to obtain A, B, C, X, Y, $Q$. Then set $M=I_{n \times n}$ and $N=I-\mathbf{Y X}$ and compute $\vec{B}_{K 1}, B_{K 2}$, $\breve{B}_{K 3}, \breve{N}$ and the matrix lifting variables $W_{1}, \ldots, W_{14}$ according to the definitions given in Section 5. Let

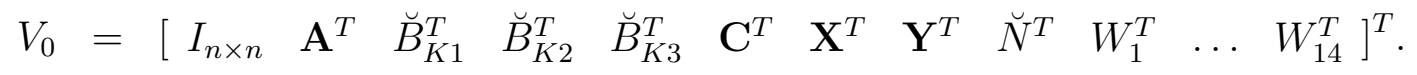

Then we set $Z=V_{0} V_{0}^{T}$ as a heuristic starting point. 


\section{An extension of the numerical procedure}

In this section, we discuss an important extension of the methodology that has been developed in preceding sections of the paper.

In Problem 3, the commutation matrix $\Theta_{K}$ is a fixed but freely specified parameter that determines the type of controller sought. However, since $A_{K}, B_{K}, C_{K}$ are all allowed to be free, it is not essential to restrict $\Theta_{K}$ to be canonical or degenerate canonical. A similarity transformation on $A_{K}$ essentially corresponds to a (matrix rescaling) of $\Theta_{K}$ (i.e. pre and post-multiplication by a non-singular matrix). Therefore it is possible to also allow $\Theta_{K}$ to be a free variable that is only restricted to be real and skew symmetric. In this setting, we do not a priori determine the type of controller that is sought (a classical, quantum, or mixed classical-quantum controller). Instead, we also seek an optimal type of controller. This leads to a modification of Problem 3 where the former restriction on $\Theta_{K}$ is removed.

Problem 6 Given a cost bound parameter $\gamma>0$, find real matrices $A_{K}, B_{K 1}, B_{K 2}, B_{K 3}$, $C_{K}$ and a skew symmetric real matrix $\Theta_{K}$ such that:

1. There exists a symmetric matrix $P>0$ satisfying (17).

2. $J_{\infty}=\operatorname{Tr}\left(\mathcal{C P C}^{T}\right)<\gamma$.

3. The resulting controller is physically realizable. That is, $A_{K}, B_{K 1}, B_{K 2}, B_{K 3}, C_{K}$ and $\Theta_{K}$ satisfy (18) and (19)

The following lemma is then immediate and shows that solving Problem 6 also solves Problem 3 .

Lemma 7 Suppose that the matrices $\hat{A}_{K}, \hat{B}_{K 1}, \hat{B}_{K 2}, \hat{B}_{K 3}, \hat{C}_{K}$ and $\hat{\Theta}_{K}$ solve Problem 6, and $\hat{\Theta}_{K}=S Z S^{T}$ for some canonical or degenerate canonical matrix $Z$ and some real invertible matrix $S$. Then the matrices

$$
A_{K}=S^{-1} \hat{A}_{K} S ; \quad B_{K i}=S^{-1} \hat{B}_{K i} \quad i=1,2,3 ; \quad C_{K}=\hat{C}_{K} S
$$

solve Problem 3 for $\Theta_{K}=Z$.

Proof. Since $\hat{\Theta}_{K}$ is real skew symmetric, we can find an invertible matrix $S$ such that $\hat{\Theta}_{K}=S Z S^{T}$ for some matrix $Z$ which is either canonical or degenerate canonical. Now, we have that

$$
\begin{aligned}
& \hat{A}_{K} \hat{\Theta}_{K}+\hat{\Theta}_{K} \hat{A}_{K}^{T}+\hat{B}_{K 1} \operatorname{diag}_{n_{1} / 2}(J) \hat{B}_{K 1}^{T}+\hat{B}_{K 2} \operatorname{diag}_{n_{2} / 2}(J) \hat{B}_{K 2}^{T}+ \\
& \quad \hat{B}_{K 3} \operatorname{diag}_{n_{3} / 2}(J) \hat{B}_{K 3}^{T}=0 \\
& \hat{B}_{K 1}=\hat{\Theta}_{K} \hat{C}_{K}^{T} \operatorname{diag}_{n_{u} / 2}(J) .
\end{aligned}
$$


After substitution of 36 and $\hat{\Theta}_{K}=S Z S^{T}$ into 37 and 38 and some algebraic manipulations, it is easily obtained that $A_{K}, B_{K}$ and $C_{K}$ satisfy

$$
\begin{aligned}
& A_{K} Z+Z A_{K}^{T}+B_{K 1} \operatorname{diag}_{n_{1} / 2}(J) B_{K 1}^{T}+B_{K 2} \operatorname{diag}_{n_{2} / 2}(J) B_{K 2}^{T}+ \\
& \quad B_{K 3} \operatorname{diag}_{n_{3} / 2}(J) B_{K 3}^{T}=0 ; \\
& B_{K 1}=Z C_{K}^{T} \operatorname{diag}_{n_{u} / 2}(J) .
\end{aligned}
$$

Moreover, the LQG cost is invariant under a similarity transformation of the controller state space matrices. Hence, these matrices solve Problem 3 with $\Theta_{K}=Z$, as claimed.

The following corollary is then obvious.

Corollary 8 Let $\Theta_{K}$ be given (canonical or degenerate canonical) and suppose that $A_{K}$, $B_{K 1}, B_{K 2}, B_{K 3}, C_{K}$ solve the standard $L Q G$ problem for a given $\gamma>0$ (i.e. (17) and $J_{\infty}<\gamma$ are satisfied), and there exists a real skew symmetric $Z$ satisfying (39) and (40). Moreover, suppose there exists a real invertible matrix $S$ such that $S Z S^{T}=\Theta_{K}$. Then the matrices $\hat{A}_{K}, \hat{B}_{K 1}, \hat{B}_{K 2}, \hat{B}_{K 3}, \hat{C}_{K}$ given by:

$$
\hat{A}_{K}=S A_{K} S^{-1} ; \quad \hat{B}_{K i}=S B_{K i} \quad i=1,2,3 ; \quad \hat{C}_{K}=C_{K} S^{-1},
$$

solve Problem 3 .

The preceding corollary says that a solution $A_{K}, B_{K}, C_{K}$ to the standard LQG problem will also solve the quantum LQG problem (Problem 3) if and only if a matrix $Z$ can be found satisfying (39) and (40) and there exists a real invertible matrix $S$ such that $S Z S^{T}=\Theta_{K}$.

It is also possible to treat Problem 6 using the rank constrained LMI procedure of Section 5. To do this, again under the simplifying assumptions $M=I$ and $N=I-\mathbf{Y X}$ (but which, as remarked earlier, can be easily removed if desired), introduce the additional variable $\Theta_{K}$ and substitute $A_{K}, B_{K i}(i=1,2,3), C_{K}$ with, respectively, $\hat{A}_{K}, \hat{B}_{K i}(i=$ 1,2,3), $\hat{C}_{K}$ (cf. Lemma 7). Then we redefine $\breve{N}=N \Theta_{K}, x=\left(x_{1}, \ldots, x_{10}\right)$ and $Z$ to be a real symmetric matrix of dimension $25 n \times 25 n$, and replace 33 with the following set of constraints:

$$
\left.\begin{array}{cc}
Z \geq 0 & \mathbf{Z}_{v_{3}, 1}-\mathbf{Z}_{x_{4}, 1} \operatorname{diag}_{n / 2}(J)=0 \\
\mathbf{Z}_{0,0}-I_{n \times n}=0 & \mathbf{Z}_{v_{4}, 1}-\mathbf{Z}_{x_{7}, 1} B=0 \\
\mathbf{Z}_{1, x_{6}}-\mathbf{Z}_{x_{6}, 1}=0 & \mathbf{Z}_{v_{5}, 1}-\mathbf{Z}_{x_{4}, 1} C-\mathbf{Z}_{x_{7}, 1} A=0 \\
\mathbf{Z}_{1, x_{7}}-\mathbf{Z}_{x_{7}, 1}=0 & \mathbf{Z}_{v_{6}, 1}-\mathbf{Z}_{x_{8}, x_{5}}=0 \\
\mathbf{Z}_{v_{1}, 1}-\mathbf{Z}_{x_{2}, 1} \operatorname{diag}_{n / 2}(J)=0 & \mathbf{Z}_{v_{7}, 1}-\mathbf{Z}_{x_{8}, x_{6}}=0 \\
\mathbf{Z}_{v_{2}, 1}-\mathbf{Z}_{x_{3}, 1} \operatorname{diag}_{n / 2}(J)=0 & \mathbf{Z}_{v_{8}, 1}-\mathbf{Z}_{x_{1}, x_{8}}=0 \\
\mathbf{Z}_{x_{9}, 1}-I_{n \times n}+\mathbf{Z}_{v_{9}, 1}=0 & \mathbf{Z}_{x_{10}, 1}+\mathbf{Z}_{1, x_{10}}=0 \\
\mathbf{Z}_{v_{9}, 1}-\mathbf{Z}_{x_{7}, x_{6}}=0 & \mathbf{Z}_{v_{13}, 1}-\mathbf{Z}_{v_{2}, x_{3}}=0 \\
\mathbf{Z}_{v_{10}, 1}-\mathbf{Z}_{v_{4}, v_{6}}=0 & \mathbf{Z}_{v_{14}, 1}-\mathbf{Z}_{v_{3}, x_{4}}=0 \\
\mathbf{Z}_{v_{11}, 1}-\mathbf{Z}_{v_{5}, v_{7}}=0 & \mathbf{Z}_{x_{8}, 1}+\mathbf{Z}_{x_{9}, x_{10}}=0 \\
\mathbf{Z}_{v_{12}, 1}-\mathbf{Z}_{v_{1}, x_{2}}=0 . &
\end{array}\right\}
$$


Here, $\mathbf{Z}_{x_{10}, 1}+\mathbf{Z}_{1, x_{10}}=0$ accounts for the requirement that $\Theta_{K}=-\Theta_{K}^{T}$ while $\mathbf{Z}_{x_{8}, 1}+\mathbf{Z}_{x_{9}, x_{10}}$ accounts for the the constraint $\breve{N}=N \Theta_{K}$. The remaining constraints of Section 5, (34) and $\operatorname{rank}(Z) \leq n$, remain the same. Note that the variable $N$ is now also an independent variable, whereas in Section $5 N$ was linearly related to $\breve{N}$ by the relation $N=\breve{N} \Theta_{K}^{-1}$ for some fixed constant $\Theta_{K}$. However, the introduction of additional free variables and constraints increase the complexity of the problem and may have a bearing on the convergence of the alternating projections algorithm. Therefore, the solver for Problem 6 may be used to complement the solver for Problem 3, and substitute for one another in case convergence fails for one of them. Furthermore, it is also necessary to develop an additional heuristic to determine a good initial guess $\Theta_{K}^{0}$ for $\Theta_{K}$. These can be topics to be considered for further research. Possible, albeit arbitrary, choices for $\Theta_{K}^{0}$ are $\Theta_{K}^{0}=0_{2 \times 2}$ or $\Theta_{K}^{0}=J$. Once $\Theta_{K}^{0}$ has been chosen, we set $N=I-\mathbf{Y X}, \breve{N}^{0}=N \Theta_{K}^{0}$ and

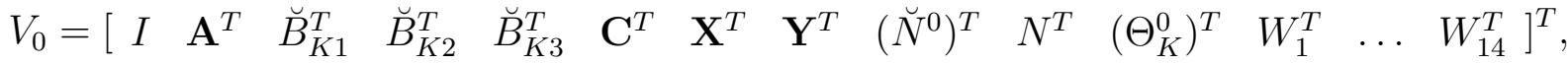

then the alternating projections algorithm can be executed by setting $Z=V_{0} V_{0}^{T}$ as the starting point.

Remark 9 It should be noted that the extended procedure should not be considered to supersede the procedure for strictly finding a quantum controller. This is because, as discussed in the Introduction, there can be circumstances where a quantum controller is desirable while the extended procedure may not return such a controller since $\Theta_{K}$ is allowed to be free.

\section{Quantum LQG control design examples}

In this section, we apply the transformation and matrix lifting technique of Section 5 to compute a fully quantum LQG controller to asymptotically stabilize a marginally stable fully quantum plant. We work in Matlab using the Yalmip prototyping environment and a solution was computed using LMIRank. The semidefinite program solver used for LMIRank is SeDuMi Version 1.1 Release 3; see [1]. Then for comparison, we also compute a classical controller that modulates a light beam to drive the plant. All computations were performed on Matlab running on an Apple Mac Pro workstation configured with two 3GHz Quad-Core Intel Xeon processors, 8GB of memory and 232.89GB hard disk capacity.

The quantum plant to be controlled is a physically realizable (cf. Section 3) fully quantum system with Hamiltonian matrix $R$ and coupling matrix $\Lambda$ (see [17] for the corresponding definitions) given by

$$
R=\frac{1}{2}\left[\begin{array}{cc}
\Delta & 0 \\
0 & \Delta
\end{array}\right] \quad \Lambda=\left[\begin{array}{cc}
\sqrt{\kappa_{1}} & 0 \\
\sqrt{\kappa_{2}} & 0 \\
\sqrt{\kappa_{3}} & 0
\end{array}\right] .
$$


Then, its dynamics are given by

$$
\begin{aligned}
& d x=\left[\begin{array}{cc}
0 & \Delta \\
-\Delta & 0
\end{array}\right] x d t+\left[\begin{array}{cc}
0 & 0 \\
0 & -2 \sqrt{k_{1}}
\end{array}\right] d u+\left[\begin{array}{cccc}
0 & 0 & 0 & 0 \\
0 & -2 \sqrt{k_{2}} & 0 & -2 \sqrt{k_{3}}
\end{array}\right]\left[\begin{array}{l}
d w_{1} \\
d w_{2}
\end{array}\right], \\
& d y=\left[\begin{array}{cc}
2 \sqrt{k_{2}} & 0 \\
0 & 0
\end{array}\right] x d t+d w_{1}
\end{aligned}
$$

with $\Delta=0.1$ and $k_{1}=k_{2}=k_{3}=10^{-2}$. This plant may be thought of as representing the scenario of an atom trapped between two mirrors of a three mirror cavity in the strong coupling limit in which the cavity dynamics are adiabatically eliminated; see [12, 8]. Note that by definition of the coupling matrix $\Lambda$, the quantum noise fields couple only to the position operator of the atom, which is also the typical setup sought in various schemes for quantum non-demolition continuous measurement of position. This particular choice of coupling results in a marginally stable plant with $A$ having two mutually conjugate eigenvalues on the imaginary axis.

\subsection{Quantum LQG controller design example I}

Let us try to asymptotically stabilize this system with another quantum system as the LQG controller. To this end we set $z=x+\xi$; i.e., $C_{z}=I_{2 \times 2}=D_{z}$. Choosing $\gamma=5.75$ and numerically solving Problem 3 following Sections 5 and 6, yields the following physically realizable controller after 1000 iterations of LMIRank (with a running time of 2944.9 seconds):

$$
\begin{aligned}
d \xi= & {\left[\begin{array}{ll}
-2.3907 & 0.8420 \\
-5.5518 & 1.9380
\end{array}\right] \xi d t+\left[\begin{array}{ll}
-0.3029 & 0.5042 \\
-0.6603 & 1.0819
\end{array}\right] d w_{K 1}+} \\
& 10^{-10}\left[\begin{array}{ll}
0.0241 & -0.0471 \\
0.0576 & -0.1136
\end{array}\right] d w_{K 2}+\left[\begin{array}{ll}
3.3626 & 2.1470 \\
7.6699 & 5.0302
\end{array}\right] d y \\
d u(t)= & {\left[\begin{array}{ll}
-1.0819 & 0.5042 \\
-0.6603 & 0.3029
\end{array}\right] x d t+d w_{K 1} }
\end{aligned}
$$

that asymptotically stabilizes the closed-loop system. The actual closed loop LQG cost achieved by this controller can be computed to be $J_{\infty}=5.7382$.

Notice that elements of $B_{K 2}$ are very small (of the order $10^{-10}$ ). This is a result that we would ideally like to have since $B_{K 2}$ is the coefficient for the quantum noise $w_{K 2}$ that only enters in the controller and does not come from the plant. This noise contributes towards the LQG cost, but since the aim is to bound this cost, it is not surprising that the algorithm finds a controller for which $B_{K 2}$ is effectively zero, in order to remove the effect of the variance of $w_{K 2}$ on the LQG cost. Up to the numerical precision of Matlab, the above numerical results give:

$$
A_{K} \Theta_{K}+\Theta_{K} A_{K}^{T}+\sum_{k=1}^{3} B_{K i} J B_{K i}^{T}=10^{-13}\left[\begin{array}{cc}
0 & 0.2896 \\
-0.2896 & 0
\end{array}\right]
$$


while dropping the quadratic term containing $B_{K 2}$ (i.e., setting $B_{K 2}=0$ ) returns an identical numerical result on the right hand side of (45). This indicates that the contribution of $B_{K 2}$ to 45 is less that the numerical precision of Matlab and we may in this case simply set $B_{K 2}=0$ to obtain a simpler controller.

\subsection{Classical LQG controller designs}

After successfully obtaining a fully quantum controller, a natural question that now arises is: Does this controller offer any improvement over a classical controller that is driven by continuous measurements (e.g., by homodyne detection; see [3]) of a quadrature of the plant output $y$ ? To answer this question, suppose now that we perform continuous measurements of one quadrature (in this case, the first element) of $y$. Thus, we replace the output $y$ in 43 by another output $y^{\prime}$ (a classical signal) given by:

$$
y^{\prime}=\left[\begin{array}{ll}
2 \sqrt{k_{2}} & 0
\end{array}\right] x d t+\left[\begin{array}{ll}
1 & 0
\end{array}\right] d w_{1} .
$$

We seek a classical controller of the form:

$$
\begin{aligned}
d \xi & =A_{K} \xi d t+B_{K} d y^{\prime} \\
\beta_{u} & =C_{K} \xi
\end{aligned}
$$

whose output modulates a light beam to produce the control signal $u$ :

$$
\begin{aligned}
d u & =\beta_{u} d t+d w_{K 1} \\
& =C_{K} \xi d t+d w_{K 1} .
\end{aligned}
$$

The optimal controller matrices $A_{K}, B_{K}, C_{K}$ can now be found by applying the standard LQG machinery (with $C_{z}$ and $D_{z}$ as before) to the following modified plant (to account for the presence of the noise $w_{K 1}$ in the controller output $u$, see a related discussion in Section 5 with $\beta_{u}$ being viewed as the "control signal":

$$
\begin{aligned}
& d x=\left[\begin{array}{cc}
0 & \Delta \\
-\Delta & 0
\end{array}\right] x d t+\left[\begin{array}{cc}
0 & 0 \\
0 & -2 \sqrt{k_{1}}
\end{array}\right] \beta_{u} d t \\
& +\left[\begin{array}{cccccc}
0 & 0 & 0 & 0 & 0 & 0 \\
0 & -2 \sqrt{k_{1}} & 0 & -2 \sqrt{k_{2}} & 0 & -2 \sqrt{k_{3}}
\end{array}\right]\left[\begin{array}{c}
d w_{K 1} \\
d w_{1} \\
d w_{2}
\end{array}\right], \\
& d y^{\prime}=\left[\begin{array}{ll}
2 \sqrt{k_{2}} & 0
\end{array}\right] x d t+\left[\begin{array}{ll}
1 & 0
\end{array}\right] d w_{1} \text {. }
\end{aligned}
$$

The optimal classical controller was then found to be:

$$
\begin{aligned}
& d \xi=\left[\begin{array}{cc}
-0.0658 & 0.1 \\
-0.1217 & -0.2
\end{array}\right] \xi d t+\left[\begin{array}{l}
0.3291 \\
0.1083
\end{array}\right] d y^{\prime} \\
& d u=\left[\begin{array}{cc}
-1 & 0 \\
0 & 1
\end{array}\right] \xi d t+d w_{K 1}
\end{aligned}
$$


while the optimal LQG cost is $J_{\infty}=4.8468$.

It turns out that cost obtained by this classical controller is lower than the cost we had previously obtained with the quantum LQG controller $\left(J_{\infty}=4.8468\right.$ in the former compared to $J_{\infty}=5.7382$ in the latter). Moreover, this may not the best performance achievable by a classical linear controller as it is also possible to perform a so-called indirect measurement of $y$ by first mixing it with an additional vacuum noise via a beam splitter [3, 10], followed by homodyne detection of the real quadrature of one output of the beamsplitter and of the imaginary quadrature of the other output, thus giving the controller noisy information about both quadratures of $y$. For examples of indirect measurement, see [17, Sections VII-C and VII-D].

To describe an indirect measurement process, suppose that the vacuum noise going into the beamsplitter is $w_{0}=\left(w_{0,1}, w_{0,2}\right)$ (in quadrature notation) and the beamsplitter divides the signals between the two ports according to the ratio $\alpha$ : $\beta$ where $\alpha^{2}+\beta^{2}=1$, $0 \leq \alpha, \beta \leq 1$. Then the classical output signal $y^{\prime \prime}$ of the indirect measurement is given by:

$$
d y^{\prime \prime}=\left[\begin{array}{cc}
\alpha & 0 \\
0 & -\beta
\end{array}\right] d y+\left[\begin{array}{cc}
\beta & 0 \\
0 & \alpha
\end{array}\right] d w_{0}
$$

Let us now seek a classical controller of the form 46 and 47 with $y^{\prime}$ replaced by $y^{\prime \prime}$ for various values of $\alpha$ between 0 and 1. Note that measurement of $y^{\prime}$ can be viewed as a special case of indirect measurement with $\alpha=1$. A plot of the LQG cost $J_{\infty}$ achieved by this controller versus the value of $\alpha$ is shown in Figure 1.
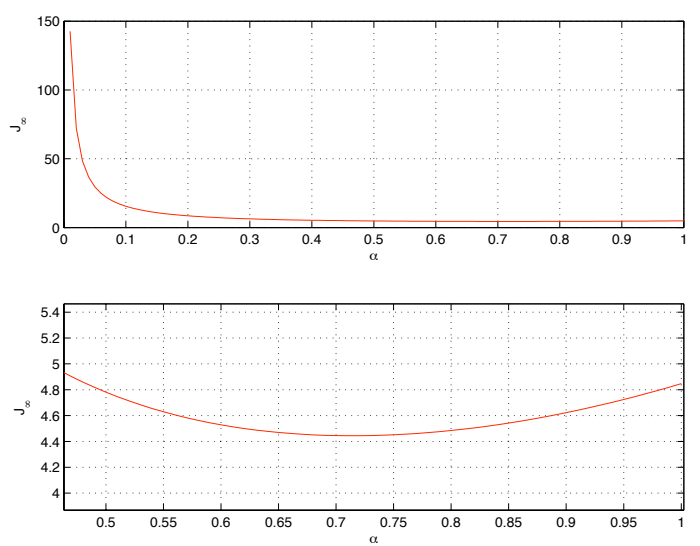

Figure 1: Plot of $J_{\infty}$ vs. $\alpha$

It can be seen that $J_{\infty}$ achieves its minimum around $\alpha=0.715$ with a value of $J_{\infty} \approx 4.4440$. This raises the question of whether there exists at all a fully quantum controller that gives a cost lower the best achievable with indirect measurements. We shall see in the next section that the answer to this is affirmative by obtaining such a fully quantum controller. 


\subsection{Quantum LQG controller design example II}

We now try to find a fully quantum controller that achieves a cost $J_{\infty}<4.444$. However, several attempts at numerically solving Problem 3 for $\Theta_{K}=J$ by alternating projections were unsuccessful with the algorithm failing to converge. Thus, we turn our attention to numerically solving Problem 6 according to the discussion in Section 7 with $\gamma=5$ and $\Theta_{K}^{0}=0_{2 \times 2}$. It turns out that the alternating projections algorithm converges (with a running time of approximately 781.87 seconds) and returns the following solution:

$$
\begin{gathered}
\Theta_{K}=\left[\begin{array}{cc}
0 & -0.1820 \\
0.1820 & 0
\end{array}\right] ; \quad \hat{A}_{K}=\left[\begin{array}{cc}
-0.2125 & 0.0666 \\
-0.3789 & 0.0257
\end{array}\right] ; \\
\hat{B}_{K 1}=\left[\begin{array}{ll}
0.0642 & -0.0547 \\
0.0480 & -0.2556
\end{array}\right] ; \quad \hat{B}_{K 2}=10^{-10}\left[\begin{array}{cc}
-0.0468 & 0.0255 \\
-0.1160 & 0.0114
\end{array}\right] ; \\
\hat{B}_{K 3}=\left[\begin{array}{ll}
0.3522 & -0.0215 \\
0.4393 & -0.0842
\end{array}\right] ; \quad \hat{C}_{K}=\left[\begin{array}{cc}
-1.4044 & 0.3008 \\
-0.2639 & 0.3526
\end{array}\right],
\end{gathered}
$$

and with the LQG cost $J_{\infty}=4.1793$.

Remark 10 It is important to note here that although the type of controller is not a priori specified, the algorithm returns $\Theta_{K}$ which corresponds (up to a similarity transformation) to a fully quantum controller, rather than a classical controller.

Since $\Theta_{K}=S J S^{T}$ with $S=\sqrt{0.182}\left[\begin{array}{ll}0 & 1 \\ 1 & 0\end{array}\right]$, then according to Lemma 7 we find the matrices $A_{K}, B_{K i}(i=1,2,3)$ and $C_{K}$ solving Problem 3 as follows:

$$
\begin{gathered}
A_{K}=\left[\begin{array}{ll}
0.0257 & -0.3789 \\
0.0666 & -0.2125
\end{array}\right] ; \quad B_{K 1}=\left[\begin{array}{ll}
0.1126 & -0.5992 \\
0.1504 & -0.1283
\end{array}\right] \\
B_{K 2}=10^{-10}\left[\begin{array}{ll}
-0.2721 & 0.0272 \\
-0.1096 & 0.0601
\end{array}\right] ; \quad B_{K 3}=\left[\begin{array}{ll}
1.0297 & -0.1974 \\
0.8255 & -0.0503
\end{array}\right] \\
C_{K}=\left[\begin{array}{ll}
0.1283 & -0.5992 \\
0.1504 & -0.1126
\end{array}\right]
\end{gathered}
$$

Note again that $B_{K 2}$ has negligibly small entries and in a similar fashion to the related discussion in Section 8.1, we may set $B_{K 2}=0$ to obtain a simpler controller. For this controller we have that the right hand side of (45) takes the numerical value $10^{-13}\left[\begin{array}{cc}0 & 0.6098 \\ -0.6098 & 0\end{array}\right]$. The fact that we were ultimately able to find a fully quantum controller that outperforms the best performance of a classical linear controller with indirect measurement (with $J_{\infty}=4.1793$ in the former compared to $J_{\infty} \approx 4.4440$ in the latter case) is very interesting and indicates that the quantum LQG controller should be considered to be more than a theoretical curiosity, but one which may potentially be of practical significance. 
It is important to note that although the controller (48) beats the class of classical linear controllers with indirect measurements, it may not beat all possible classical controllers. For example, there may exist classical non-linear controllers that could perform just as well or better.

Remark 11 Here we do not discuss the physical realizations of fully quantum controllers such as (44) and (48) in the laboratory using currently available quantum optical components. This is addressed in the work of [27] on a network synthesis theory for dynamical quantum optical networks that parallels that of electrical network synthesis theory.

\section{Conclusions}

In this paper, we have formulated a quantum LQG problem that allows the possibility for the controller to be another quantum system. In general, this problem is a polynomial matrix programming problem that can be systematically converted to a rank constrained LMI problem. To solve the problem, we propose a numerical procedure based on an alternating projections algorithm and an extension of this procedure for the case where the type of controller is not a priori specified. In two examples, we consider the problem of stabilization of a marginally stable quantum plant and successfully compute some fully quantum LQG controllers that achieve this goal. We show that for the fully classical controller schemes considered herein (in which continuous measurements are performed on the output of the plant), a fully quantum LQG controller can be found which gives an improved level of performance.

It is important to emphasize that the quantum LQG problem posed here cannot be solved by simply applying the standard LQG methodology, since the resulting controller may not be physically realizable (i.e., the controller system matrices are not guaranteed to satisfy (18) and (19)). In fact, unlike the classical LQG problem that can be reformulated as a convex LMI problem, the quantum LQG problem is in general computationally hard.

For future investigation, theoretical aspects of the quantum LQG problem posed herein deserve further study. At the moment, system theoretic conditions for the existence of a solution to this problem are not known. Another avenue which is of importance for future investigation is the practical implementation of quantum LQG controllers and, indeed, general linear quantum controllers in the laboratory.

Acknowledgement 12 The first author thanks $R$. Orsi for discussions on rank constrained LMI problems and the LMIRank software, V. P. Belavkin for discussions on quantum linear systems and dynamic programming, and J. Löfberg for correspondences on the Yalmip software.

\section{References}

[1] Advanced Optimization Lab, McMaster University . SeDuMi v1.1R3. October 2006. 
[2] M. A. Armen, J. K. Au, J. K. Stockton, A. C. Doherty, and H. Mabuchi. Adaptive homodyne measurement of optical phase. Phys. Rev. A, 89(13):133602, 2002.

[3] H.A. Bachor and T.C. Ralph. A Guide to Experiments in Quantum Optics. WileyVCH, Weinheim, Germany, second edition, 2004.

[4] V.P. Belavkin. On the theory of controlling observable quantum systems. Automation and Remote Control, 44(2):178-188, 1983.

[5] V.P. Belavkin. Continuous non-demolition observation, quantum filtering and optimal estimation. In Quantum Aspects of Optical Communication, volume 45 of Lecture notes in Physics, pages 131-145. Springer, Berlin, 1991.

[6] L. Bouten, R. van Handel, and M. R. James. An introduction to quantum filtering. SIAM J. Control Optim., 46:2199-2241, 2007.

[7] S. Boyd and L. Vanderberghe. Semidefinite programming relaxations of non-convex problems in control and combinatorial optimization. In A. Paulraj, V. Roywhcowdhury, and C. Schaper, editors, Communications, Computation, Control and Signal Processing - A Tribute to Thomas Kailath. Kluwer Academic Publishers, New York, 1997.

[8] A.C. Doherty and K. Jacobs. Feedback-control of quantum systems using continuous state-estimation. Phys. Rev. A, 60:2700, 1999. quant-ph/9812004.

[9] S. C. Edwards and V. P. Belavkin. Optimal quantum filtering and quantum feedback control. August 2005.

[10] C. Gardiner and P. Zoller. Quantum Noise: A Handbook of Markovian and Non-Markovian Quantum Stochastic Methods with Applications to Quantum Optics. Springer Series in Synergetics. Springer, 2 edition, 2000.

[11] J. M. Geremia, J. K. Stockton, and H. Mabuchi. Real-time quantum feedback control of atomic spin squeezing. Phys. Rev. A, 304:270-273, 2004.

[12] J. Gough and R. van Handel. Singular perturbation of quantum stochastic differential equations with coupling through an oscillator mode. J. Stat. Phys., 127(3):575-607, 2007.

[13] D. Henrion and J. B. Lasserre. Convergent relaxations of polynomial matrix inequalities and static output feedback. IEEE Trans. Automat. Contr., 51(2):192-202, 2006.

[14] C. W. J. Hol and C. Scherer. Sum of squares relaxations for polynomial semidefinite programming. In Proc. 16th International Symp. Mathemarical Theory and Networks (MTNS) 2004 (Leuven, Belgium, July 5-9, 2004). 2004. 
[15] R. L. Hudson and K. R. Parthasarathy. Quantum Ito's formula and stochastic evolution. Commun. Math. Phys., 93:301-323, 1984.

[16] B. C. Jacobs, T. B. Pittman, and J. D. Franson. Quantum relays and noise suppression using linear optics. Phys. Rev. A, 66:052307, 2002.

[17] M. R. James, H. I. Nurdin, and I. R. Petersen. $H^{\infty}$ control of linear quantum stochastic systems. IEEE Trans. Automat. Contr., 53(8):1787-1803, 2008.

[18] E. Knill, R. Laflamme, and G.J. Milburn. A scheme for efficient quantum computation with linear optics. Nature, 409:46-52, 2001.

[19] M. Kojima. Sums of squares relaxations of polynomial semidefinite programs. Technical Report B-397, Dept. Math. Comp. Sci., Tokyo Institute of Technology, Tokyo, Japan, 2003.

[20] P. Kok, C. P. Williams, and J. P. Dowling. The construction of a quantum repeater with linear optics. Phys. Rev. A, 68:022301, 2003.

[21] J. B. Lasserre. Global optimization with polynomials and the problem of moments. SIAM J. Optimization, 11(3):796-817, 2001.

[22] G. Linblad. Brownian motion of harmonic oscillators: Existence of a subdynamics. J. Math. Phys., 39(5):2763-2780, 1998.

[23] J. Löfberg. Yalmip : A toolbox for modeling and optimization in MATLAB. In Proceedings of the CACSD Conference, Taipei, Taiwan, 2004.

[24] E. Merzbacher. Quantum Mechanics. Wiley, New York, third edition, 1998.

[25] Y. Nesterov and A. Nemirovskii. Interior-point polynomial algorithms in convex programming. SIAM, Philadelphia, 1994.

[26] M.A. Nielsen and I.L. Chuang. Quantum Computation and Quantum Information. Cambridge University Press, Cambridge, 2000.

[27] H. I. Nurdin, M. R. James, and A. C. Doherty. Network synthesis of linear dynamical quantum stochastic systems. provisionally acceptable for publication subject to some minor revisions, SIAM Journal on Control and Optimization (2009), June 2008.

[28] R. Orsi. LMIRank: Software for rank constrained LMI problems. 2005.

[29] R. Orsi, U. Helmke, and J. B. Moore. A Newton-like method for solving rank constrained linear matrix inequalities. Automatica J. IFAC, 42(11):1875-1882, 2006. Extended version available at R. Orsi's homepage.

[30] K.R. Parthasarathy. An Introduction to Quantum Stochastic Calculus. Birkhauser, Berlin, 1992. 
[31] C. Scherer, P. Gahinet, and M. Chilali. Multiobjective output-feedback control via LMI optimization. IEEE Trans. Automat. Contr., 42(7):896-911, 1997.

[32] A.J. Shaiju, I.R. Petersen, and M.R. James. Guaranteed cost LQG control of uncertain linear stochastic quantum systems. In Proceedings of the 2007 American Control Conference, New York, July 2007.

[33] N. Z. Shor. Dual quadratic estimates in polynomial and boolean programming. Ann. Oper. Res., 25:163-168, 1990.

[34] The Mathworks, Inc. Optimization Toolbox 3: User's Guide. The Mathworks, Inc, 9th printing edition, September 2007.

[35] H. Wiseman and G.J. Milburn. Quantum theory of optical feedback via homodyne detection. Phys. Rev. Lett., 70(5):548-551, 1993. 